\title{
Rickettsial infections in Sri Lanka: yesterday, today and tomorrow
}

\author{
Ranjan Premaratna ${ }^{1}$ \\ Journal of the Ceylon College of Physicians, 2011, 42, 11-15
}

In late 2004, a 45-year old school teacher from Divulapitiya presented with an undiagnosed fever headache and body aches for 10 days duration. On admission, she was conscious and rational and had no other clinical features to suggest a specific aetiological diagnosis. On the second day of admission, she became increasingly drowsy, restless and agitated. A working diagnosis of encephalitis, meningo-encephalitis or cerebral malaria was made and was commenced on triple therapy with iv quinine, ceftriaxone and acyclovir. However, she continued to deteriorate despite treatment and the next day the bystander complained that she could not hear. On examination, she was stony deaf and had rapid clinical deterioration and investigations including an MRI scan of the brain were not revealing any abnormality. However, on careful examination, a lesion mimicking an eschar was noted hidden in the skin folds of right axilla. A prompt diagnosis of scrub typhus was made and she was commenced on iv chloramphenicol and doxycycline via an naso-gastric tube. She had a dramatic recovery over the next 24 hours, and became afebrile in 48 hours. She started to hear by that time and had a complete clinical recovery in 72 hours with objective hearing improvement in two weeks. Her illness was later confirmed serologically as scrub typhus caused by Orientia tsutsugamushi at the Rickettsial Zoonoses Branch (RZB), Centers for Disease Control and Prevention (CDC), Atlanta, Georgia, USA.

\section{Rickettsioses}

Rickettsioses are caused by a group of pleomorphic, gram negative, bacteria like intracellular micro-organisms. Although most rickettsial organisms are non-pathogenic to humans, some of them are known to cause febrile illnesses when transmitted accidently via blood sucking arthropods such as ticks, fleas and mites. The historical classification of rickettsioses was based on the intracellular location of the parasite, optimum temperature for growth, type of vector and cross reaction of patient's sera to three

\footnotetext{
${ }^{1}$ Department of Medicine, Faculty of Medicine, University of Kelaniya, Sri Lanka.
}

different antigens of bacillus Proteus. However, with recent research based on molecular techniques and sequence based taxonomy they have been reclassified.

The early history of typhus fever dates back to 1546s when Fracastorius sufficiently described it as a different disease entity. However, it is thought as to whether the aetiology of the great epidemic of Athens described vividly by Thucydides in $430 \mathrm{BC}$ was due to typhus fever. Typhus fever has a close link with the history of military efforts as it had played a decisive role many battles such as the siege in Granada in 1489 and defeat of French in Naples in 1528. Later Napoleon's campaign in 1812 had ended up in disaster because of typhus. During World War 1, between 1918 and 1922 approximately 30 million contracted typhus in Russia and estimated 3 million had died.

The most renowned scientists who carried out research on rickettsioses are Howard Taylor Ricketts (1871-1910), attached to the University of Chicago and did extensive studies on Rocky Mountain spotted fever and Stanislaus Matthias Von Prowazek (1875-1915) a Czech protozoologist who studied the aetiology of typhus. However, both died of contracting the illness in the course of their studies and later in honour of their contributions, the aetiological agent of louse borne typhus was named as Rickettsia prowazekii. The transmission of typhus by body lice was conclusively shown by Charles Jules Henri Nicolle (1866-1936), the director of Pasteur Institute in Tunis in 1909. This discovery had significant bearings on the control of typhus fevers, thus he was awarded the Nobel Prize in 1928.

\section{History of Rickettsioses in Sri Lanka}

In Sri Lanka, then Ceylon, the history of typhus fever dates back to 1930-1940s during massive military efforts in the Eastern theatre. The descriptions of the illness is a result of investigations of fever among the military deployed in South East Asia, therefore, it is likely that typhus fever existed in Ceylon long before the war efforts. Amongst such descriptions South East Asian War Office document a dramatic outbreak of Scrub typhus in over 750 East African and British troops during a 4 day military exercise in Embilipitiya carrying a high morbidity. The first recorded reference 
on rickettsial infections in Ceylon dates back to 1937 in the opening paragraph of a short report published by Nicholls. According to that, L.B.E.Seneviratne in 1937 had examined 434 patient blood samples in provincial hospitals by the Weil-Felix reaction and 6 were found to be positive for scrub typhus. Then in 1938, Cyril F Fernando described two patients with fever for two weeks and a skin rash from Nuwara Eliya, confirmed having tropical typhus (Murine typhus). Seneviratne of the Bacteriological Institute, Colombo and Wolf, the City Microbiologist inoculated blood of the second patient into Guinea pigs and demonstrated fever, splenomegaly and scrotal swelling in them. His research concluded that the source of infection of the patient to be rat fleas as he was a salesman of a dry fish store in Pettah, Colombo. He later demonstrated abundance of typhus infested rats transported in Indian vessels anchored in Colombo port in surrounding granaries, warehouses and shops. After Fernando's report, Wijerama published two cases of tropical typhus in 1938 followed by Nicholls a case of Tsutsugamushi fever (Rural typhus) in Ceylon in the BMJ in 1940. In 1946, Jayawickreme and Niles have studied the role of trombiculid mites in the transmission of rural typhus. However, even as late as 1956, physicians in Ceylon seldom suspected typhus when dealing with PUO. The first large scale sero-epidemiological survey was done in 1972 among hospital staff and patients in Colombo and found a crude prevalence rate of $6 \%$ for scrub typhus. The occurrence of SFG rickettsioses in Sri Lanka was first reported in 1994 by Rajaratnam et al and Vasanthatilaka and Senanayake reported eight cases of murine typhus in 1994. However, the first case series on rickettsioses confirmed IFA assay was published by Kularatne et al in 2003 from the central province.

\section{Research carried out and publications}

Since the presentation of the patient with encephalitis and deafness described above, we collected clinical data of patients who were suspected of having acute rickettsioses. They were later confirmed having rickettsial aetiology by IFA (immuno-fluroscent antibody testing). After my presentation of these cases at the Annual Academic Sessions of the American Society of Rickettsiology in Colorado, USA, I was invited for laboratory training in rickettsial diseases diagnostics at Rickettsial Zoonoses Branch, CDC, Atlanta, Georgia, USA. On my return I established Rickettsial Disease Diagnostic and Research Laboratory (RDDRL) at the Faculty of Medicine, University of Kelaniya. The initial collaborative work with CDC and later after the establishment of RDDRL resulted in several research projects and publications as highlighted below.

\section{Acute hearing impairment due to scrub typhus; a forgotten complication of a re-emerging disease. Clinical Infectious Diseases}

Since the admission of the first patient who developed deafness with scrub typhus, from November 2003 - May 2004, there were several patients who presented with similar clinical illness with varying degrees of severity and all of them responded to oral doxycycline treatment. They were later confirmed serologically as having scrub typhus caused by Orientia tsutsugamushi at the Rickettsial Zoonoses Branch (RZB), Centers for Disease Control and Prevention (CDC), Atlanta, Georgia, USA.

\begin{abstract}
"We described 6 patients with scrub typhus who presented with acute hearing loss, a forgotten complication of this reemerging disease. They were admitted with fever of 10-14 days' duration and had clinical evidence of deafness and pneumonitis. Five patients had eschars, which prompted the diagnosis of typhus fever and led to early institution of treatment. Deafness has been described as a clue to the diagnosis of scrub typhus; awareness of this symptom facilitated early diagnosis in 4 of 5 patients who recovered. Acute hearing loss or hearing impairment in a febrile patient should arouse strong suspicion of scrub typhus".
\end{abstract}

2. Rickettsial infections and their clinical presentations in Western Province of Sri Lanka: a hospital based study. International Journal of Infectious Diseases

Clinical details and laboratory parameters of 31 suspected rickettsioses were collected for a period of one year commencing from November 2002. A total of 31 patients (22 female, mean age 52 years (SD 5.6)) fulfilled our case definitions and were further investigated. Paired blood samples were obtained at least two weeks apart from 11 (35\%) patients. All patients presented as sporadic cases and were living an average of $10 \mathrm{~km}$ (range 4 - $25 \mathrm{~km}$ ) from the hospital. Of these patients, 19 (61\%) were living in urban areas and 12 (39\%) were living in semi-urban areas. They were living in houses with cement floors and either tile or asbestos sheet roofing. All of them claimed that there were rats in the vicinity, and all patients had engaged in some activity in the garden surrounding their house. None of them had visited scrub jungle areas, slept on the floor or bare ground, or visited areas outside their hometown during the preceding three months.

Out of the 31 patients with possible rickettsioses, 29 (94\%) fell into the categories of confirmed, presumptive, or exposed cases of acute rickettsial 
infections (Scrub typhus was diagnosed in 19 (66\%), spotted fever group in eight (28\%)). Early acute infection or past exposure was suggested in $2(7 \%)$ cases; cross-reactivity of antigens or past exposure to one or more species was suggested in 9 (31\%). Seventeen out of 19 (89\%) patients with scrub typhus had eschars. Nine out of 29 (32\%) patients had a discrete erythematous papular rash: seven caused by spotted fever group, two by scrub typhus.

The most striking feature among the uncomplicated cases was that the patients did not feel very ill when afebrile. However, the bouts of fever were associated with chills, severe headache, and body aches. Patients without a rash seem to have had more frequent, high grade, intermittent fever than those with a rash. Nine out of 29 patients (32\%) had a discrete erythematous papular rash mainly distributed over the extremities, involving palms and soles; seven were caused by spotted fever group Rickettsia spp and two by $O$. tsutsugamushi. The rashes were most prominent at the time when the patients were febrile. There were no haemorrhages or tissue necrosis associated with the rashes. However, rashes were often accompanied by a patch of redness in the lateral limbus of each eye, which again was more prominent when the patient was febrile.

The severe complications among rickettsioses included myocarditis, pneumonitis, encephalitis. The mean duration of illness at the time of admission in patients who developed complications was 12.0 days (SD 1.4) compared to 9.2 days (SD 3.5) for those who had no complications ( $p<0.05)$. Myocarditis, seen in five patients, was characterized by transient arrhythmia (atrial fibrillation), low blood pressure, and a transient reduction in ejection fraction. One patient demonstrated apical hypokinesia on echocardiography, which subsequently disappeared. Patients with pneumonitis had a dry cough associated with coarse crepitations. There was no reduction in capillary oxygen saturation except in the two seriously ill patients, who had minimum $\mathrm{O}_{2}$ saturations of 94 and $92 \mathrm{KPa}$, respectively. Of those with hearing impairment, three had bilateral high tone deafness, which progressed to complete deafness in less than 24 hours; clinical recovery with treatment was dramatic, and objective improvement was proportionate to the initial degree of deafness. Features of encephalitis included high frequency coarse tremors of extremities associated with abnormal lateral head movements and rapid oscillations of the eyes in all directions. The latter manifestations were seen in the more severe cases. With one exception, all patients had complete symptomatic recovery within 48 hours of commencement of treatment with oral doxycycline.
Two patients who had severe myocarditis, encephalitis, and pneumonitis were treated with intravenous chloramphenicol in addition to doxycycline. One patient died despite this treatment. Following this study, we concluded that in a region representing the low country wet zone of Sri Lanka, the main rickettsial agent seems to be Orientia tsutsugamushi. Delay in diagnosis may result in complications. All species responded well to current treatment.

\section{Contribution of rickettsioses in Sri Lankan patients with fever who responded to empirical doxycycline treatment. Transactions of the Royal Society of Tropical Medicine and Hygiene}

Over a 2-year period from 2005, 28 patients with febrile illness, with neither definitive diagnosis nor improvement with another treatment for 7 days after admission to hospital, but who later responded to empirical treatment with doxycycline $100 \mathrm{mg}$ bid for 7 days were further investigated, retrospectively. All these patients had presented to the Professorial Medical Unit, Colombo North Teaching Hospital, Ragama, Sri Lanka. A detailed history was obtained covering animal contact, tick bites and visits to jungles or scrub lands, and a careful clinical examination excluded eschars, vasculitic rashes, Iymphadenopathy or hepatosplenomegaly. To investigate for rickettsioses, we used a preserved $1 \mathrm{ml}$ sample of serum obtained at the time of admission as part of the broader fever study. The samples were preserved at $-20^{\circ} \mathrm{C}$ until they were analyzed. Indirect fluorescent antibody assays (IFA) were carried out using rickettsial antigens prepared from cell culture grown rickettsiae: $R$. conorii (Malish), $R$. typhi (Wilmington), and O. tsutsugamushi (Karp). $\operatorname{IgM}$ and IgG antibodies were detected using fluorescein-conjugated goat anti-human $\lg M$ and $\lg G(\square)$ respectively (KPL, Inc., Gaithersburg, MD, USA). Sera were screened at 1:32, and positive samples were titred to the endpoint of 1:1024 for IgG and IgM. To assay IgM, samples positive for IgG were separated using spin columns. As only five patients presented for followup after one month, a diagnosis of rickettsial infection was defined on the basis of their presentation with a febrile illness, rapid defeverence to doxycycline, and the presence of a species specific IFA IgM titre of $\geq 1 / 64$ combined with a positive IgG2 titre of $>1: 256^{1}$.

All 28 patients (15 male, 13 female, mean age 32.5 years (SD: 9.2)) were from the Western Province (low country, wet zone). The mean duration of fever at admission and when doxycycline was given were 8.1 days (SD 3.1) and 15.29 days (SD 1.6) respectively. The mean (SD) white cel count was $9.3 \times 10^{9} / 1$ (2.3) with 65\% (7.57) neutrophils. The mean (SD) CRP and ESR were $39.7 \mathrm{mg} / \mathrm{l}$ (24.5) and $47 \mathrm{~mm}$ first hour (20.9) 
respectively. Of the 28 patients, 11 were diagnosed with acute SFG rickettsioses and 10 with $O$. tsutsugamushi infections. None were positive for $\mathrm{R}$ typhi. One of two patients with encephalitis and the single patient with erythema nodosum had high titres for SFG rickettsioses. Only seven were negative for all tests.

Our results indicated that, patients with fever, with no definitive diagnosis 7 days after hospitalization, having normal white cell counts and moderate elevations of CRP and ESR, who responded to empirical doxycycline, were suffering from a rickettsial infection. Thus, empirical treatment with anti-rickettsial antibiotics, such as doxycycline, is justified in patients with undiagnosed fever who have no clinical features or investigation results suggesting another aetiology, in settings where rickettsial infections are endemic or re-emerging. The main advantage of such therapy is that, if the illness is rickettsial in origin, the clinical response is dramatic even when serious complications are present, and morbidity and mortality may be greatly reduced. It may also help to curtail costs by reducing hospital stay and avoiding extensive investigations that are required for non-resolving fevers.

With this study, we concluded that empirical use of anti-rickettsial antibiotics to be helpful in the management of patients with febrile illness in settings where rickettsial infections are endemic or re-emerging and diagnostic facilities are inadequate. However, establishment of proper diagnostic facilities is urgently required to understand the varied clinical patterns and changing epidemiology of these infections.

Thereafter, we described several clinical presentations of rickettsioses.

\section{Unusual pancytopenia secondary to haemo- phagocytosis syndrome in rickettsioses. Transactions of the Royal Society of Tropical Medicine and Hygiene}

We reported two patients who presented with a long duration febrile illness associated with pancytopenia. Both of them had evidence of hypercellular marrow with haemophagocytosis. One was confirmed as having Orientia tsutsugamushi infection, and that was the first reported case of scrub typhus causing pancytopenia. The other patient was infected by spotted fever group rickettsioses. Both of them had a rapid haematological recovery with antirickettsial antibiotics. With these case reports, we highlighted the importance of considering rickettsial infections in patients with such clinical presentations, especially in areas where these infections are endemic or re-emerging. Empirical use of anti-rickettsial antibiotics in such situations could be beneficial, when facilities to diagnose rickettsial diseases are not readily available.

5. Rickettsioses presenting as major joint arthritis and erythema nodosum: description of four patients. Clinical Rheumatology 2009

Erythema nodosum and aseptic arthritis are recognized associations of rickettsial infections. However, they usually present with a febrile illness rather than with severe arthritis. We reported three patients who presented with incapacitating major joint arthritis and one who presented with severe spondyloarthropathy in addition to major joint arthritis due to serologically confirmed Orientia tsutsugamushi and Rickettsia conorii infections. All of them had erythema nodosum and low-grade fever. They had rapid clinical response to doxycycline.

\section{Scrub typhus mimicking enteric fever: a report of three patients. Transactions of the Royal Society of Tropical Medicine and Hygiene}

We reported three patients who presented with fever and late onset diarrhoea mimicking enteric fever. All three patients were diagnosed with an Orientia tsutsugamushi infection and responded dramatically to doxycycline treatment. We suggested that clinicians practicing in rickettsial disease endemic areas should be made aware of similar clinical presentations in order to prevent morbidity and mortality associated with rickettsioses.

\section{Rickettsioses in Sri Lanka: future activities}

As for future research in rickettsioses, in order to improve early diagnosis, and therefore to reduce morbidity and mortality due to rickettsioses, PCR based diagnositics should be made available in Sri Lanka. Such facilities would also help to identify vector species and related dynamics and, various other animals harboring rickettssial agents, and therefore issues associated with transmission of rickettsioses in the country.

\section{Publications}

1. Premaratna R, Chandrasena TGAN, Dassanayake AS, Lofftis AD, Gregory DA, de Silva HJ. Acute hearing impairment due to scrub typhus; a forgotten complication of a re-emerging disease. Clinical Infectious Disease 2006; 42: e6-8. Epub 2005 Nov 29.

2. Premaratna $R$, Loftis $A D$, Chandrasena TGAN, Dasch GA, de Silva HJ. Rickettsial infections and their Clinical Presentations in Western Province 
of Sri Lanka: a hospital based study. International Journal of Infectious Disease 2008; 12: 198-202. Epub 2007 Sep 27.

3. Premaratna R, Rajapakse RPVJ, Chandrasena TGAN, Nanayakkara DM, Bandara NKBKRGW, Kularatna SAM, Eremeeva ME, Dasch GA, de Silva HJ. Contribution of rickettsioses in Sri Lankan patients with fever who responded to empirical doxycycline treatment. Transactions of the Royal Society of Tropical Medicine and Hygiene 2009; 103: 961-3. Epub 2009 May 15.

4. Premaratna R, Chandrasena TG, Rajapakse RP, Eremeeva ME, Dasch GA, Bandara NK, de Silva $\mathrm{HJ}$. Rickettsioses presenting as major joint arthritis and erythema nodosum: description of four patients. Clinical Rheumatology 2009; 28: 867-8. Epub 2009 Mar 25.

5. Premaratna R, Williams HS, Chandrasena TG, Rajapakse RP, Kularatna SA, de Silva HJ. Unusual pancytopenia secondary to haemophagocytosis syndrome in rickettsioses. Transactions of the Royal Society of Tropical Medicine and Hygiene 2009; 103: 961-3. Epub 2009 May 15.

6. Premaratna R, Nawasiwatte BM, Niriella MA, Chandrasena TG, Bandara NK, Rajapakse RP, de Silva HJ. Scrub typhus mimicking enteric fever: a report of three patients. Transactions of the Royal Society of Tropical Medicine and Hygiene 2010; 104: 309-10. Epub 2009 Sep 17. 